\title{
A Rare Sweat Gland Tumour- Immunohistochemical Features
}

\author{
MIHAELA-CRISTINA PRODEA ${ }^{1}$, FLORICA SANDRU2*, ECATERINA CARPENCO ${ }^{3}$, PUSA NELA GAJ E4, \\ IOANA DELIA HORHAT ${ }^{1}$, AMALIA RALUCA CEAUSU ${ }^{4}$, NARCISA MEDERLE ${ }^{5}$, OVIDIU ALEXANDRU MEDERLE ${ }^{4,6}$ \\ ${ }^{1}$ Victor Babes University of Medicine and Pharmacy, Department of ENT, 2 EftimieMurgu Sq., 300041, Timisoara, Romania \\ ${ }^{2}$ Carol Davila University of Medicine and Pharmacy, Department of Dermatovenerology, Elias Emergency University Hospital, 8 \\ Eroii Sanitari Blvd., 050471, Bucharest, Romania \\ ${ }^{3} \mathrm{~N}$. Testemitanu University of Medicine and Pharmacy, Department of Histology, 165 Stefan cel Mare si Sfant Blvd., MD-2004, \\ Chisinau, Republica Moldova \\ ${ }^{4}$ Victor Babes University of Medicine and Pharmacy, Department of Microscopic Morphology/ Histology, Angiogenesis Research \\ Center Timisoara, 2 Eftimie Murgu Sq., 300041, Timisoara, Romania \\ ${ }^{5}$ Banat's University of Agricultural Sciences and Veterinary Medicine King Michael I of Romania, Parasitology/Mycology Department, \\ 119, CaleaAradului, 300645, Timisoara, Romania. \\ ${ }^{6}$ Municipal County Hospital, Emergency Department, 24 Gh. Dima Str., 300079, Timisoara, Romania
}

\begin{abstract}
The main types of normal sweat glands are eccrine and apocrine. The papillaryeccrine adenoma is a rare, benign, sweat gland tumor. The clinical aspect is a slow growing solitary dermal nodule on the extremities, less frequently involving the trunk or face. Here it was described a case of papillary eccrine adenoma developed in the scapular-humeral region in a 72 years old man. The $K 5+/ K 7+/ K 8 / 18+/ K 19+/$ SMA+/ S100+ immunohistochemical profile and the morphological aspects support the diagnostic of papillary eccrine adenoma. The immunohistochemical profile is useful for the differential diagnostic and may increase the diagnosis accuracy.
\end{abstract}

Keywords: papillary eccrine adenoma, immunohistochemistry

Two types of normal sweat glands can be identified: eccrine and apocrine. The first type is distributed over the entire skin surface and open to the epidermis. Apocrine glands are found in the axilla, mons pubis, perianal and periareolar regions, and open to the follicular infundibulum. The sweat glands are composed of 3 segments: the intraepidermal(eccrine)/intrainfundibular (apocrine) duct, the intradermal duct, and the secretory portion. The secretory portion of eccrine glands has a narrow tubular structure composed of pale inner secretory cells and outer myoepithelial cells, whereas apocrine glands shows large tubules composed of eosinophilic secretory cells and peripheral myoepithelial cells [1].

Classification of cutaneous sweat gland adnexal lesions according to the current concept of the predominant accepted origin, consists of: eccrine and apocrine (mixed origin): hidrocystoma, apocrine/eccrine nevus, tubulopapillaryhidradenoma (including papillary eccrine adenoma and tubular apocrine adenoma), chondroidsyringoma; eccrine origin: poroma, hidradenoma, spiradenoma, cylindroma, syringometaplasia, syringoma; apocrine origin: syringocystadenomapapilliferum, hidradenomapapilliferum; composite/ mixed cutaneous adnexal tumours [2].

Rulon and Helwig $[3,4]$ described for the first time, in 1977, the papillary eccrine adenoma as a rare, benign, sweat gland tumor. The commonly clinical aspect is a slow growing solitary dermal nodule on the extremities, less frequently involving the trunk or face.

We described here a case of papillary eccrine adenoma that developed in the scapular-humeral region in a 72years oldman.

\section{Experimental part}

A 72-year- old manpresented with an ulcerated nodular tumor on the left scapular-humeral region. The lesion measured $2-3 \mathrm{~cm}$ in diameter. The patient had a simple excision of the nodule.

The excised mass was fixed in 10\% buffered formalin, paraffin embedded, cut into $3 \mu \mathrm{m}$ sections, and stained with hematoxylin and eosin (HE) and immunohistochemical techniques. The entire immunohistochemical technique was performed with Leica Bond Max (Leica Biosystem, Newcastle Upon Tyne, UK). The following primary antibodies were used: keratin 5 (clone XM 26), keratin 7 (clone RN7), keratin 8/18 (clone 5D3), keratin 19 (clone b170), keratin 20 (clone PW 31), smooth muscle actin (clone alpha-sm1), mast cell tryptase (clone 10D11), ki67 (clone MM1), chromogranin A (clone 5H7), S100 protein (polyclonal). All of these were ready to use antibodies, from Leica Bond, Leica Biosystem, Newcastle Upon Tyne, UK. The incubation time was 30 min and succeed the pretreatment and peroxidase blocking steps. The Bond Polymer Refine Detection System was used for visualization, 3,3 diaminobenzidine as chromogen and hematoxylin for counterstain.

Morphological staining showed nodular proliferation with uniform cells. Minor atypia was noticed. The papillary projections were found in the inner part of the nodules. $A$ slightly acidophilic secretion, which in some areas suggests aberrant sebum storage was noticed. Focal hyalinized zones were present. Some of the morphological features are showed in the figures 1 and 2 .

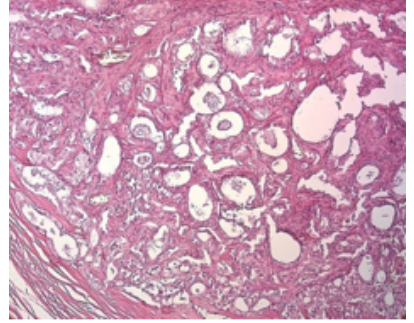

Fig. 1.The papillary projections in the inner part of the nodules, HE staining, ob.X20

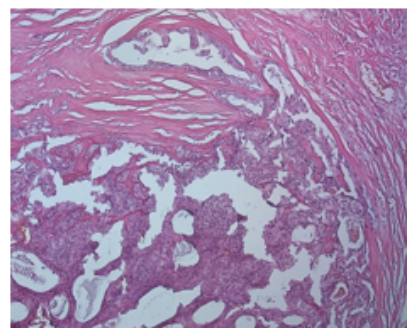

Fig. 2.The papillary projections in the inner part of the nodules, HE staining ob.X40 
Immunohistochemical staining revealed the following cytokeratin profile: $\mathrm{K} 5+/ \mathrm{K} 7+/ \mathrm{K} 8 / 18+/ \mathrm{K} 19+/ \mathrm{K} 20-$. Keratin 5 was expressed in the basal cells with granular cytoplasmic pattern. Keratin 7 was intense positive, with luminal profile (fig. 3). K8/18 was intensely expressed in the glandular proliferation (fig. 4, 5). Keratin 19 had a homogeneous distribution pattern, with moderate intensity (fig. 6).

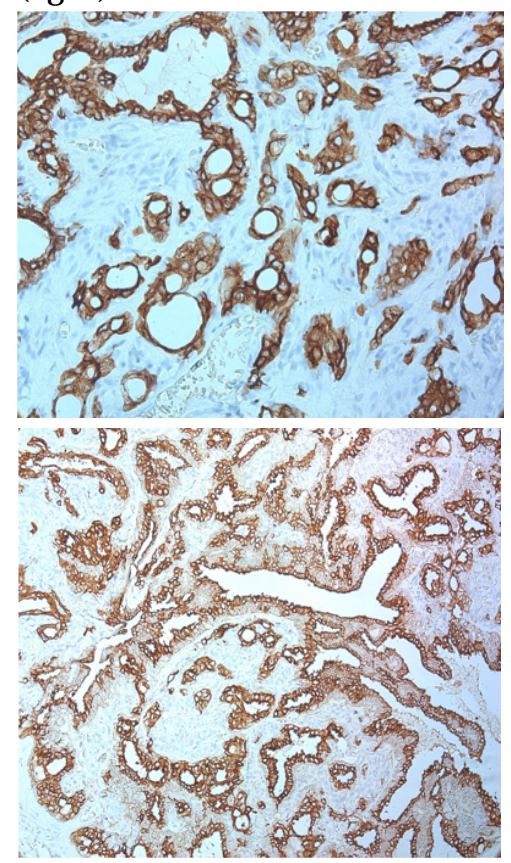

Fig.3.Keratin

7immunoexpression, ob.X20

Fig.4. Keratin 8/18 immunoexpression, ob.X20

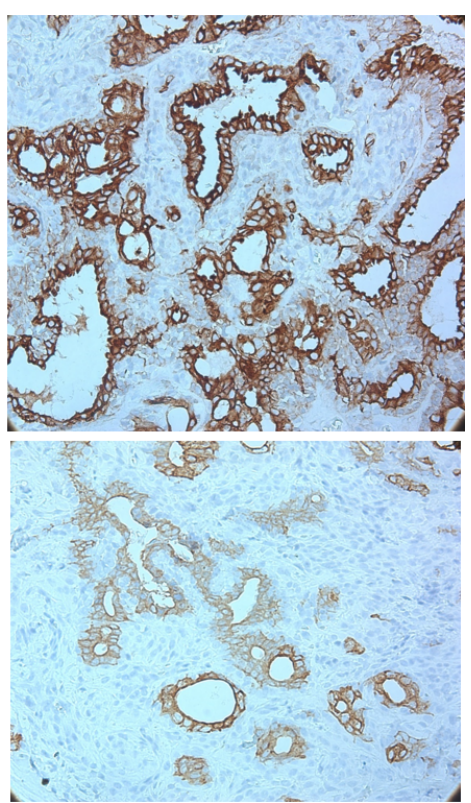

Fig.5. Keratin 8/18 immunoexpression ob.X40

Fig.6.Keratin 19 had a homogeneous distribution pattern, with moderate intensity

Smooth muscle actin expression was noticed intralobular, around glandular structure. Myoepithelial cells were positive, with cytoplasmic expression (fig. 7, 8).A high number of mast cells in the interlobular stroma of the tumor and few cells in the intralobular stroma was found

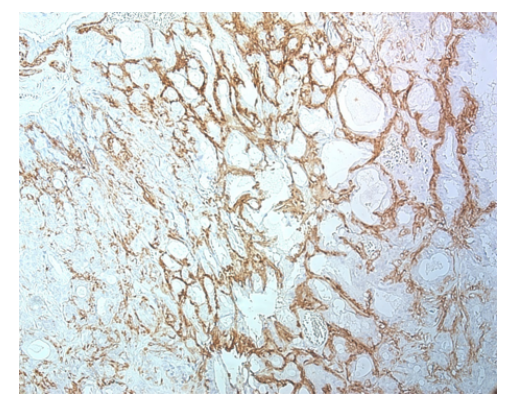

Fig.7.Expression of smooth muscle actin, ob. X20 (fig. 9,10). The immunohistochemical pattern was granular cytoplasmic. S100 was positive in the proliferative and periglandular cells (fig. 11).

Chromogranin A was negative. Ki 67 nuclear expression indicate a proliferative rate between $5-6 \%$. The immunohistochemical features are showed in the figure 3,4 .

Smooth muscle actin expression was noticed intralobular, around glandular structure. Myoepithelial cells were positive, with cytoplasmic expression (fig. 5, 6).

A high number of mast cells in the interlobular stroma of the tumor and few cells in the intralobular stromawas found (fig. 7, 8). The immunohistochemical pattern was granular cytoplasmic.

S100 was positive in the proliferative and periglandular cells (fig. 11).

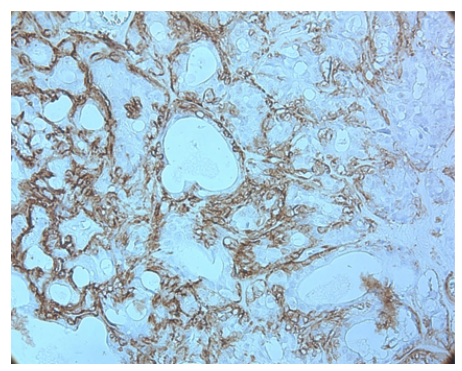

Fig.8. Expression of smooth muscle actin, ob. X40

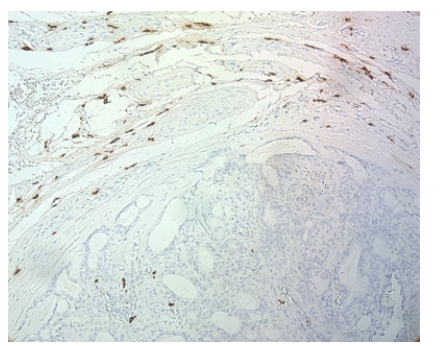

Fig.9. Mast cell distribution pattern, in the intra and peritumoral zones ob.X20

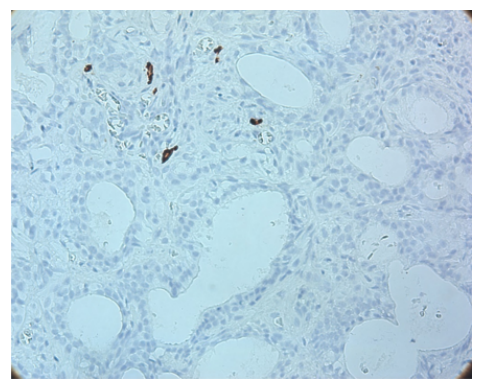

Fig.10.Mast cell distribution pattern, in the peritumoral and intratumoral areas, ob.X 40

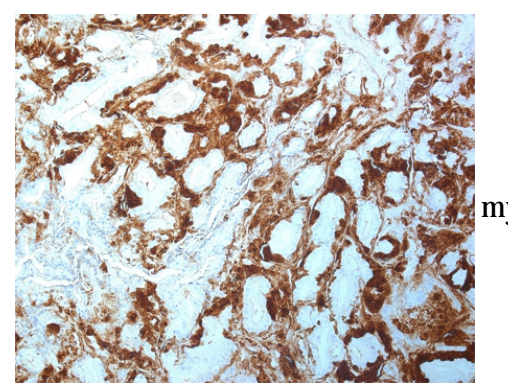

Fig.11. S100

immunoexpression, myoepithelial positive cells, ob. X 40

\section{Results and discussions}

The term tubulopapillaryhidradenoma describes benign sweat gland tumors, characterized by combining ductal as well as apocrine and eccrine glandular differentiation which include both papillary eccrine adenoma and tubular apocrine adenoma [5]. Tubular apocrine adenoma usually presents on the scalp as superficial dermal nodule and shares the histological features of papillary eccrine adenoma, except that the latter has a peripheral myoepithelial cell layer. The S100+ / CEA+ / EMA+ phenotype indicated eccrine origin of papillary eccrine adenoma. In our case, the $\mathrm{K} 5+/ \mathrm{K} 7+/ \mathrm{K} 8 / 18+/ \mathrm{K} 19+/$ 
SMA+/S100+ immunohistochemical profile together with the morphological aspects support the diagnostic of papillary eccrine adenoma.

Around 50 cases of papillary eccrine adenoma were reported in the English Literature so far [6]. It was showed that papillary eccrine adenoma occurs between 9-78years [7]. Our case, 72 years old man falls within this age range, but doesn't respect the preferred occurrence in women, with a ratio male to female 7:27.

The differential diagnostic can be made with other benign tumours or with aggressive types of neoplasm. In the first category, the immunohistochemical profile ( $\mathrm{K} 5+$ ) K7-/K8/18+/ K19-/K20-/ S100-) may indicates a sebaceous origin of adenoma. The tubular apocrine adenoma is characterized by: localization frequently on the scalp, decapitation secretion, shorter papillary projections into the lumen, lack connections with epidermis, the presence of plasma cells and tendence to co-occur with syringocystadenomapapilliferum [8].

For the second category, the differential diagnosis may include the adenocarcinomas and basal cell carcinoma of eccrine differentiation. The main characteristics of adenocarcinomas are: atypical mitosis, pleomorphism, vascular and perineural invasion, tendency for local recurrence [9]. The similarities between papillary eccrine adenoma and basal cell carcinoma of eccrine differentiation are: tubules consisting of one or two layers of cuboidal cells, surrounded by fibrous stroma, intraluminally eosinophilic granular material. These carcinomas consist cystic, alveolar and cribriform zones and had an aggressive potential. Unlike the papillary eccrine adenomas, the carcinomas didn't contain intracysticpapillations [10].

\section{Conclusions}

Despite of typical histological features of this slow growing benign tumour, it can resemble other cutaneous neoplasms. It can be associated with diagnostic challenges and immunohistochemistry profile- $\mathrm{K} 5+1$ $\mathrm{K} 7+/ \mathrm{K} 8 / 18+/ \mathrm{K} 19+/ \mathrm{SMA}+/ \mathrm{S} 100+$ may increase the diagnosis accuracy.
Acknowledgements. The authors are grateful to Victor Babes University of Medicine and Pharmacy Timisoara, Romania, Research Grant No.PIIC5-TC-2017-08 - 770/17.01.2017. Also we would like to thanks to ours histotechnologists Patricia Berzava and CiprianOnica for their excellent technical support.

\section{References}

1. NISHIDA H, DAA T, KASHIMA K; ARAKANE M, URABE S, YOSHIKAWA Y, GAMACHI A, YOKOYAMA S, KIT (CD117) expression in benign and malignant sweat gland tumors, The American Journal of Dermatopathology 2015; 37(12): 898-905.

2.OBAIDAT NA,ALSAAD KO,GHAZARIAN D, Skin adnexal neoplasmspart 2: An approach to tumours of cutaneous sweat glands, J ClinPathol 2007;60:145-159.

3. RULON, DB, HELWIG EB, Papillary eccrine adenoma, Archives of dermatology1977; 113(5): 596-598.

4. KURTZ DH, FINNELL JA, MEHLER AS, Papillary eccrine adenoma of the heel: A case report, The Journal of foot and ankle surgery2000; 39(4): 249-252.

5.SHARMA A, KUNDAL R, SINGH H, Tubular Apocrine Adenoma - A Case Report of Rare Sweat Gland Tumor, Annals of International Medical and Dental Research 2017;

3(3): $44-45$.

6.MATHEWS M, KUMAR A, FROMOWITZ A, SIMONHOFF B, MAROULES M, AKMAL A, Papillary Eccrine Adenoma: A Recent Review of Literature, American Journal of Medical Case Reports 2016; 4(9): 304-309.

7. MIZUOKA H, SENZAKI H, SHIKATA N, UEMURA Y, TSUBURA A, Papillary eccrine adenoma: Immunohistochemical study and literature review, J ournal of cutaneous pathology 1998; 25(1): 5964.

8. ICHIKAWA E, OKABE S, URNEBAYASHI Y, IIJ IMA S, OTSUKA F, WATANABE S, Papillary eccrine adenoma: immunohistochemical studies of keratin expression. Journal of cutaneous pathology, 1997; 24(9): 564-570.

9. SEXTON, M, MAIZE J C,Papillary eccrine adenoma: a light microscopic and immunohistochemical study, Journal of the American Academy of Dermatology 1988; 18(5): 1114-1120. 10. JACKSON EM, COOK J, Mohs micrographic surgery of a papillary eccrine adenoma, Dermatologic surgery 2002; 28(12): 1168-1172.

$\overline{\text { Manuscript received:22.08.2018 }}$ 